\title{
Comparison of platelet-rich plasma and extracorporeal shock wave therapy in patients with chronic lateral epicondylitis: A prospective, randomized-controlled study
}

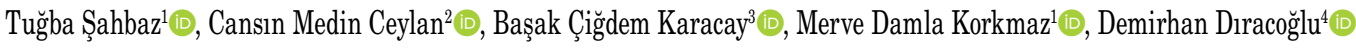 \\ 1Department of Physical Medicine and Rehabilitation, University of Health Science, Kanuni Sultan Süleyman Training and Research Hospital, Istanbul, Turkey \\ ${ }^{2}$ Department of Physical Medicine and Rehabilitation, University of Health Sciences, Istanbul Physical Therapy Rehabilitation Training and Research Hospital, Istanbul, Turkey \\ ${ }^{3}$ Department of Physical Medicine and Rehabilitation, Yozgat Yerkoy State Hospital, Yozgat, Turkey \\ ${ }^{4}$ Department of Physical Medicine and Rehabilitation, Istanbul University, Istanbul Faculty of Medicine, Istanbul, Turkey
}

Received: April 14, 2020 Accepted: January 13, 2021 Published online: December 01, 2021

\begin{abstract}
Objectives: In this study, we aimed to investigate the effectiveness or comparative therapeutic superiority of exercise, extracorporeal shock wave therapy (ESWT), and platelet-rich plasma (PRP) on pain, grip strength and functional activities in chronic lateral epicondylitis (LE).

Patients and methods: Between January 2016 and February 2017, a total of 74 patients (14 males, 60 females; mean age; $49.7 \pm 7.6$ years, range, 26 to 60 years) with chronic LE were included in this prospective, randomized-controlled study. All patients received stretching and eccentric strengthening exercises for three months. The patients were divided into three groups. The first group (Exercises group, $\mathrm{n}=24$ ) was given home exercises. The second group (ESWT+Exercises group, $\mathrm{n}=25$ ) received one session of ESWT added once a week for three weeks. The third group ( $\mathrm{PRP}+$ Exercises group, $\mathrm{n}=25$ ) received one session of PRP in addition to the exercise program. All patients were evaluated for pain by Visual Analog Scale (VAS), for functionality by Disabilities of Arm, Shoulder and Hand (DASH) questionnaire and Patient-Rated Tennis Elbow Evaluation (PRTEE), handgrip strength by a dynamometer, and extensor tendon thickness by ultrasonography (USG) at baseline and at one, two, three, and six months.

Results: A significant improvement was found in the VAS, DASH, PRTEE, handgrip strength values at six months compared to between in all groups $(p<0.001)$. Extensor tendon thickness as assessed by USG indicated no significant difference ( $>0.05)$. Regarding the VAS activity levels, there was a significant difference in the PRP+Exercises group compared to the Exercises group at six months of follow-up ( $<<0.001)$. The decrease in the DASH scores during six-month follow-up was significantly higher in the PRP+Exercises group compared to the Exercises group ( $\mathrm{p}=0.004)$. For the PRTEE scores at six months, the PRP+Exercises group showed a statistically significant improvement than both Exercises $(\mathrm{p}<0.001)$ and ESWT+Exercises $(\mathrm{p}=0.007)$ groups.

Conclusion: In the treatment of chronic LE, PRP combined with exercise seems to be superior to exercise or ESWT in terms of pain and functionality in chronic LE patients.
\end{abstract}

Keywords: Chronic, exercise, extracorporeal shock wave therapy, lateral epicondylitis, platelet-rich plasma.

Lateral epicondylitis (LE) is the most common cause of elbow pain in adults. ${ }^{[1]}$ It affects nearly 1 to $3 \%$ of the adult population. It is commonly observed in the fifth and sixth decades of life and is seen equally between both sexes. It is primarily caused by repetitive excessive wrist extension, leading to chronic pain syndrome. ${ }^{[2,3]}$

There is no consensus on the effectiveness or comparative therapeutic superiority of the current treatment modalities. ${ }^{[4]}$ The effect of exercise is

Corresponding author: Tuğba Şahbaz, MD. SBÜ Kanuni Sultan Süleyman Ĕgitim ve Araştırma Hastanesi Fizik Tedavi ve Rehabilitasyon Kliniği, 34303 Küçükçekmece, İstanbul, Türkiye. e-mail: piskint@gmail.com 
prominent in the literature. ${ }^{[5]}$ The number and size of the collagen fibrils increase by exercise treatment. ${ }^{[6]}$ No specific exercise program has been described for LE; however, exercise trainings which elevate the loading tolerance of the extensor tendons have been emphasized. ${ }^{[7]}$

Extracorporeal shock wave therapy (ESWT) has been used in the treatment of complications in the musculoskeletal system such as plantar fasciitis, LE, shoulder tendonitis and patellar tendonitis for two decades. ${ }^{[8,9]}$ The essential principle of ESWT is conversion of a chronic condition to an acute condition. Its possible mechanism of action involves increasing blood circulation, induction of the required immune response, and stimulation of healing response by causing new tissue damage. ${ }^{[10]}$

Platelet-rich plasma(PRP) is the plasma component which is obtained by centrifugation of whole blood and contains a higher platelet concentration than whole blood. ${ }^{[1]]}$ The presence of numerous growth factors in its content has introduced the use of PRP injections in treatment of various diseases in the musculoskeletal system. Histopathological evidence has demonstrated that deficiency of repair mechanisms, rather than inflammation, plays a role in the pathological process of chronic soft tissue degeneration. ${ }^{[11,12]}$ Growth factors that are considered to be effective in the healing process may have a potential use in the treatment of the lesion via local injection owing to their effect on tendon and cartilage tissue regeneration. ${ }^{[12-14]}$

In the literature, there is only a limited number of studies comparing these modalities. In the present study, we aimed to investigate the effectiveness or comparative therapeutic superiority of exercise, PRP, and ESWT in patients with chronic LE.

\section{PATIENTS AND METHODS}

This single center, prospective, randomizedcontrolled study was conducted at Istanbul University, Istanbul Faculty of Medicine, Department of Physical Medicine and Rehabilitation between January 2016 and February 2017. A total of 81 patients over 18 years of age who were admitted to our clinic with elbow complaints for more than three months, despite application of other conventional treatment methods were included. Patients were diagnosed with LE based on physical examination using the Mills test, Maudsley test, and Cozen's test. Two positive results without another pathology as confirmed by X-ray were considered in favor of LE. The patients with a medical history of injection treatment within the last three months, history of previous surgery or shock wave therapy on the treatment site, coagulation problems, peripheral vasculopathy, complex regional pain syndrome, local infections, malignancy, arthritis, direct trauma to elbow and pregnancy were excluded from the study. Finally, 74 patients (14 males, 60 females; mean age; $49.7 \pm 7.6$ years, range, 26 to 60 years) were included in the study. The study flow chart is shown in Figure 1. A written informed consent was obtained from each patient. The study protocol was approved by the Istanbul University, Istanbul Faculty of Medicine Ethics Committee (Date-No: 2014-1894). The study was conducted in accordance with the principles of the Declaration of Helsinki.

The patients were randomly divided into three groups. The first group (Exercises group, $\mathrm{n}=24$ ) was followed under an exercise program involving two sets of stretching and eccentric strengthening exercises every day for three months. The second group (ESWT+Exercises group, $\mathrm{n}=25$ ) was followed under a treatment regimen including three sessions of ESWT once a week for three weeks in addition to exercise treatment. The third group (PRP+Exercises group, $\mathrm{n}=25$ ) was followed under a treatment regimen involving one session of PRP in addition to exercise treatment.

Stretching wrist extensors and eccentric strengthening exercises were given daily, two sets of 10 reps for three months, as the exercise program. Stretching exercises were performed as static stretching keeping the wrist at complete flexion. Eccentric strengthening exercises were started by elbow at full extension and dorsiflexed wrist making fist. It is performed by bringing the wrist to flexion in 6 to 8 sec by applying submaximal endurance from upward using the hand on the healthy side. Additionally, all the patients were trained about the activities to avoid. The exercise program was followed by weekly telephone calls.

In our study, radial ESWT was performed in three sessions once a week using the ShockMaster 500 device (GymnaUniphy NV, Bilzen, Belgium). A gel was applied on the region with the highest pain and tenderness at the epicondylar area, and radial shock waves were applied in circular motions using a standard $15-\mathrm{mm}$ applicator. The ESWT was applied at 2,000 pulses of $10 \mathrm{~Hz}$ frequency and 1.8 bar of air pressure for each session. 
For PRP therapy, $20 \mathrm{~mL}$ venous blood was drawn into $2 \mathrm{Na}$-citrate test tubes. Two tubes were inserted into the centrifuge opposite each other and centrifuged at 2,000 $\mathrm{rpm}$ for $10 \mathrm{~min}$. The buffy coat and plasma obtained by centrifugation were taken into another test tube, which was activated by $10 \%$ calcium chloride. After activation, the test tubes were centrifuged at $4,000 \mathrm{rpm}$ for $5 \mathrm{~min}$. The obtained plasma was taken into an injector without mixing with the precipitate below. The elbow of the patient was positioned at $45^{\circ}$ flexion, while the forearm was in pronation. A $3 \mathrm{~mL}$ PRP was injected radially.

\section{Evaluation and follow-up}

Physical examination, pain assessment, functional assessment and ultrasonography (USG) were performed at baseline and at one, two, three, and six months. All ESWT and PRP applications and evaluation of the results were carried out by a single physician experienced in injection.

Levels of pain at activity, rest and night were questioned by the Visual Analog Scale (VAS, 0-10 points). In our study, basic grip strength was assessed using the Jamar hydraulic hand dynamometer (Sammons Preston Rolyan, Bolingbrook, IL, USA) which was found to have a high validity and reliability in many studies and accepted as the gold standard, since it is recommended by the American Society of Hand Therapists (ASHT). ${ }^{[15]}$ The patients were requested to squeeze the hand dynamometer, until feeling discomfort to determine the painless grip strength. ${ }^{[16]}$ The first test was performed with the elbow in $90^{\circ}$ flexion and the second test with the elbow in extension position. ${ }^{[16,17]}$

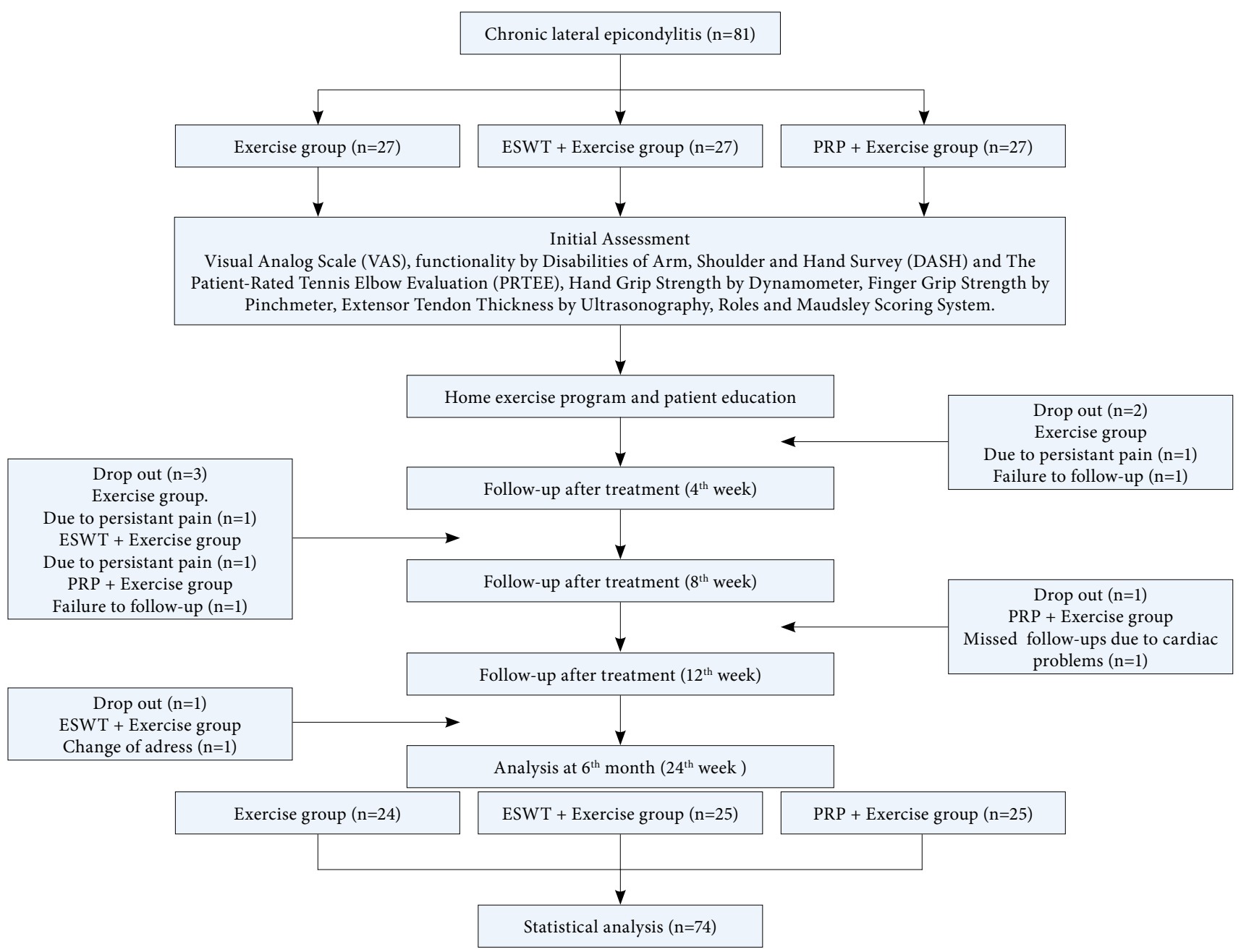

Figure 1. Study flow chart.

ESWT: Extracorporeal shock wave therapy; PRP: Platelet-rich plasma. 
The pinch meter (Baseline ${ }^{\oplus}$ Hydraulic Pinch Gauge, Irvington, NY, USA) was used to assess the finger grip strength. The measurements were performed as the key aspect, and the mean value was recorded in $\mathrm{kg}$ after repeating for three times.

The Disabilities of the Arm, Shoulder and Hand (DASH) questionnaire that was originally developed by the American Academy of Orthopedic Surgeons (AAOS) in 1994 and measures physical disabilities and symptoms in all the upper limb disorders ${ }^{[18]}$ and the Patient-Rated Tennis Elbow Evaluation (PRTEE) test, a special assessment form for the patients with $\mathrm{LE},{ }^{[19]}$ were used during all follow-up examinations before and after the treatment. In DASH and PRTEE evaluations, a value between $0-100$ is obtained as the total score, and higher scores indicate severe disability.

All USG examinations were performed using the MyLab ${ }^{\mathrm{TM}} 60$ device (Esaote S.p.A., Genoa, Italy) with a linear transducer at 6 to $18 \mathrm{MHz}$ frequency bandwidth by an experienced physiatrist who has been performing musculoskeletal USG for more than 10 years. The patients were positioned with elbow flexed at $90^{\circ}$ and pronated. The transducer of the USG was longitudinally placed on the radial surface of the elbow, and the thickness of the common extensor tendon was measured in $\mathrm{mm} .{ }^{[20]}$

The primary endpoint was the improvement in pain from baseline to six months, as assessed by the VAS (the minimum clinically important difference on the $10-\mathrm{cm}$ scale was $3-\mathrm{cm}$ ).

The patient satisfaction after the treatment was assessed using the Roles and Maudsley Scale (RMS). ${ }^{[21]}$

\section{Statistical analysis}

The study power and sample size calculation were performed using the $G^{*}$ Power version 3.0.10 (Heinrich-Heine-Universität Düsseldorf, Düsseldorf, Germany) to ensure the adequate sample size for one-way analysis of variance (ANOVA): repeated measure between factors test. To obtain a power of 0.80 [ $\alpha$ (Type I error) was 0.05 and $\beta$ (Type II error) was 0.20 , a mean effect size $(0.30)$ and three intervention groups with five repetitions], appropriate total sample size was 69 ( $n=23$ in each group) for this study. Considering $20 \%$ drop-out rate, a total of $81 \quad(n=27$ in each group) patients were included in the study. The patients who met the inclusion criteria were randomized using a computer-based randomization program (www.randomizer.org).
Statistical analysis was performed using the IBM SPSS for Windows version 22.0 software (IBM Corp., Armonk, NY, USA). Descriptive data were expressed in mean \pm standard deviation (SD), median (min-max) or number and frequency, where applicable. The distributions of the variables were controlled by Shapiro-Wilk test. Categorical variables were compared using the chi-square test. The intra-group comparisons were carried out using the Friedman test. The post-hoc analysis with the Wilcoxon signed-rank tests was conducted using the Bonferroni correction at a significance level of $<0.017$. Repeated measures ANOVA was used where appropriate, followed by the Bonferroni post-hoc test. Inter-group comparisons were performed by the Kruskal-Wallis with the Dunn's post-hoc test for the difference between pre-treatment and post-treatment (at six month). A $p$ value of $<0.05$ was considered statistically significant.

\section{RESULTS}

Among a total 74 patients included in the study, there was no significant difference in the demographic characteristics and baseline measurements of all groups ( $\mathrm{p}>0.05)$ (Table 1).

A statistically significant improvement was found in terms of VAS, DASH, PRTEE, handgrip strength (Jamar dynamometer), and finger grip strength (pinch meter) between baseline and the post-treatment sixth month measurements in all three groups $(p<0.001)$. However, the extensor tendon thickness by USG indicated no statistically significant difference at six months in the intra-group comparisons in all three groups ( $p>0.05$ ).

A statistically significant improvement was observed in VAS activity and DASH scores starting from the first month in all three groups compared to baseline $(\mathrm{p}<0.05)$. The PRTEE scores were also significantly improved starting from the first month in the PRP and ESWT groups, while an improvement was seen after two months in the exercise group.

The painless grip values of handgrip strength at flexion position showed a significant improvement after one month in the PRP group and two months in the ESWT and Exercise groups. Painless grip values of handgrip strength at extension position also showed a significant improvement in all groups after two months.

For inter-group comparisons, the differences between pre-treatment and post-treatment sixth-month values were calculated. No significant 


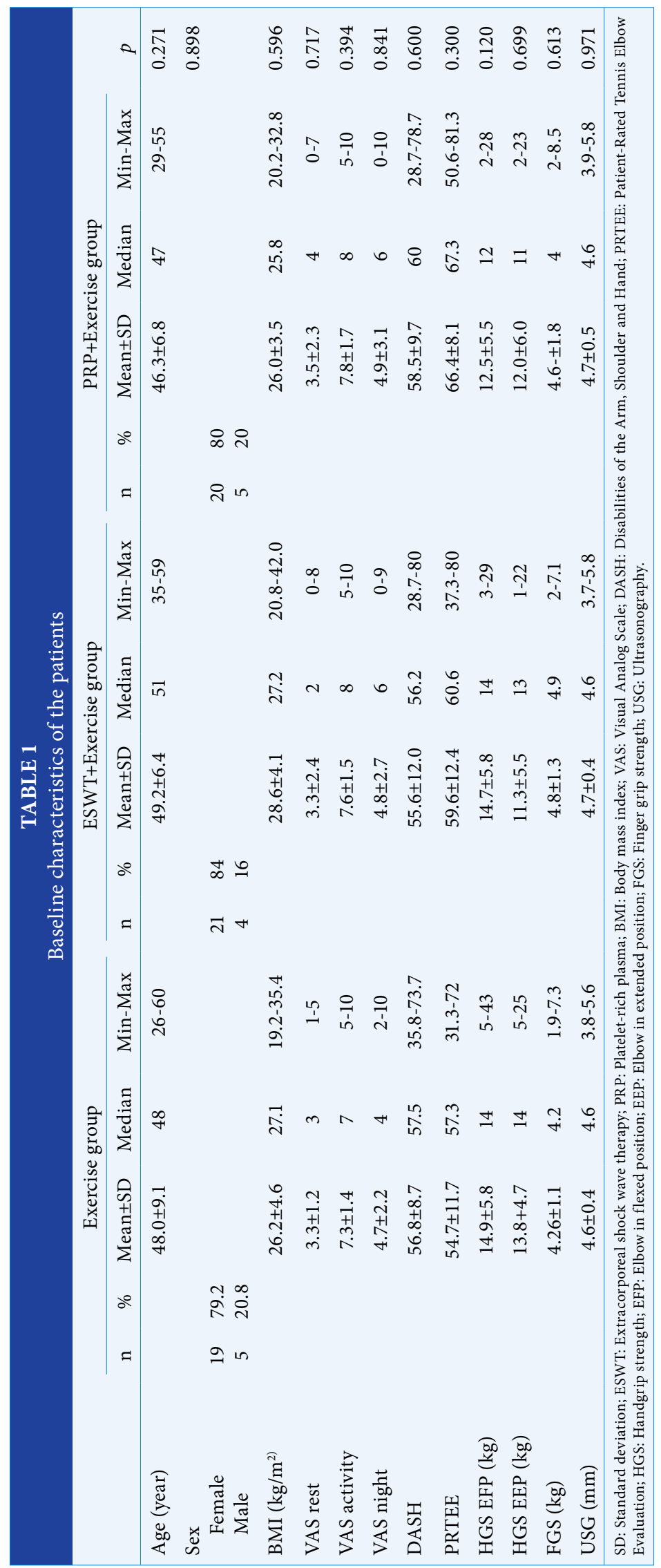



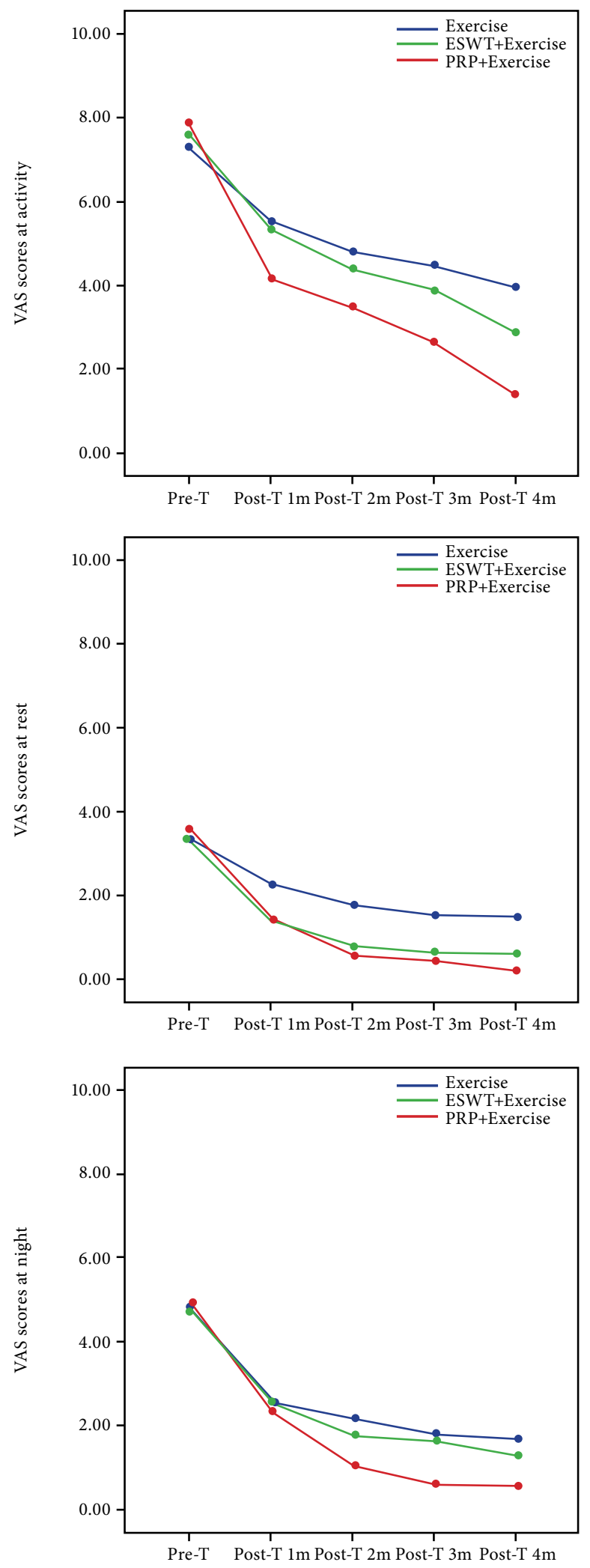

Figure 2. VAS score changes during follow-ups according to groups.

VAS: Visual Analog Scale, Pre-T: Pre-treatment; Post-T: Post-treatment; ESWT: Extracorporeal shock wave therapy; PRP: Platelet-rich plasma. difference was observed among the treatment groups in terms of resting and nighttime parameters of VAS at six months of follow-up $(p>0.05)$. On the other hand, regarding the VAS activity, there was a significant difference in the PRP+Exercise group compared to the Exercise group at six months of follow-up $(\mathrm{p}<0.001)$ (Figure 2).

Considering the number of patients with VAS activity levels 1 and below 1, there were two patients at one month, three patients at three months, and four patients at six months after the treatment in the exercise group. In the ESWT group, there were one patient at one month and four patients at six months. In the PRP group, there were four patients at one month, six patients at three months, and nine patients at six months with VAS activity level 1 and below 1 .

A statistically significant difference was noted between the PRP+Exercise and Exercise groups regarding the DASH scores at the post-treatment sixth month compared to baseline $(\mathrm{p}=0.004)$. In the comparison of the treatment groups in terms of PRTEE scores, a statistically significant difference was found between both the PRP+Exercise and Exercise groups $(\mathrm{p}<0.001)$ and between PRP+Exercise and ESWT+Exercise groups $(\mathrm{p}=0.007)$ at six months of follow-up (Table 2).

The comparison among the groups in terms of the difference in painless grip values of handgrip strength at both flexion and extension positions between baseline and post-treatment sixth month revealed a statistically significant difference only between the PRP+Exercise and Exercise treatment groups (flexion position $\mathrm{p}=0.007$ vs. extension position $\mathrm{p}=0.005)$. The comparison among the groups in terms of the difference in painless grip values of finger grip strength between baseline and post-treatment sixth month also revealed a statistically significant difference between the PRP+Exercise and Exercise treatment groups $(\mathrm{p}=0.009)$ (Table 3).

Comparison of the difference in extensor tendon thickness via USG measurements between pre-treatment and post-treatment sixth month indicated no statistically significant difference among the three groups ( $>0.05$ ) (Table 4).

The RMS scores for the patient satisfaction revealed a statistically significant difference in the PRP+Exercise group compared to the Exercise group $(\mathrm{p}=0.028)$ (Table 5). 


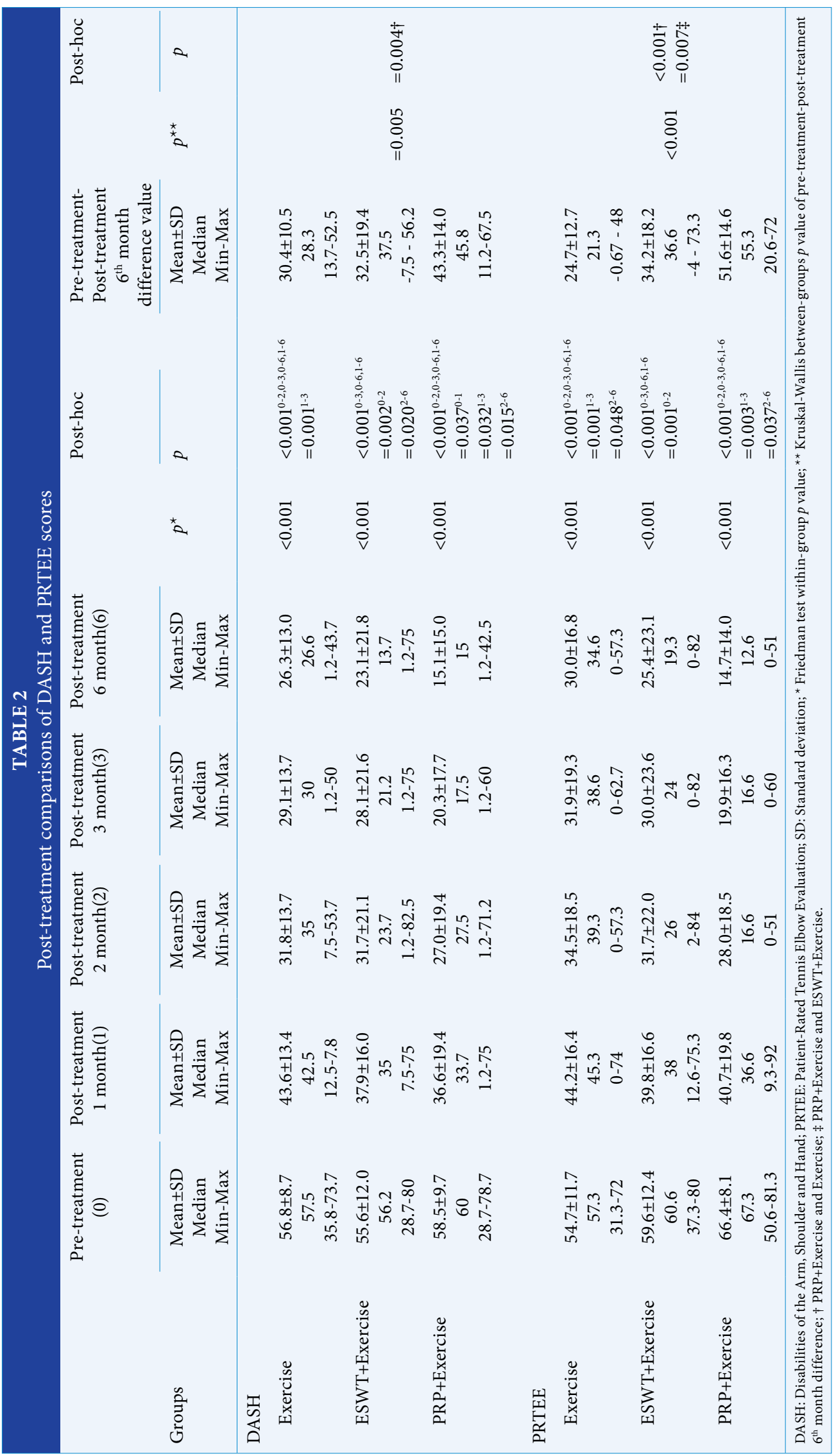




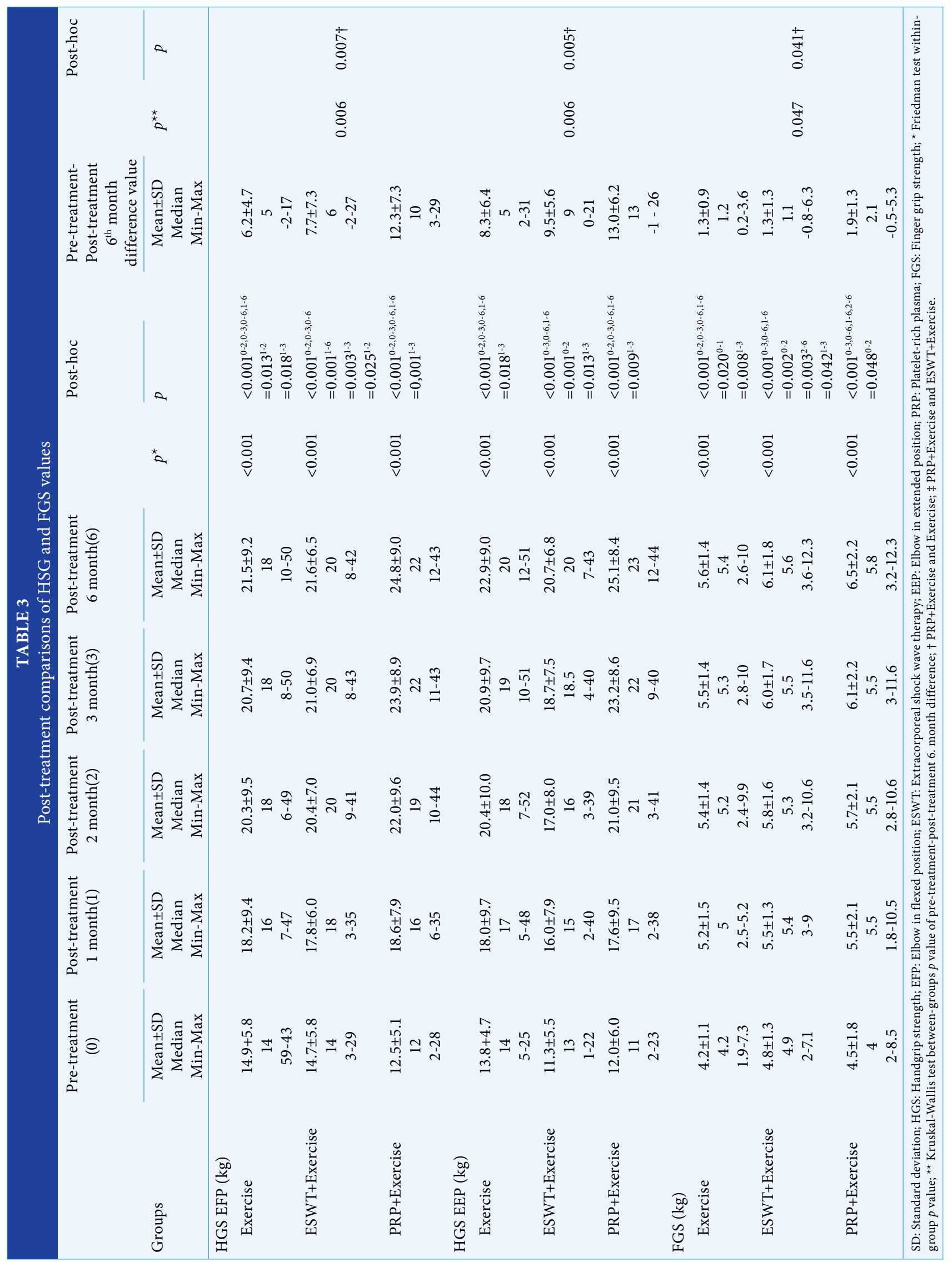




\begin{tabular}{|c|c|c|c|c|c|c|}
\hline \multicolumn{7}{|c|}{$\begin{array}{c}\text { TABLE } 4 \\
\text { Post-treatment comparisons of USG values }\end{array}$} \\
\hline & Pre-treatment & $\begin{array}{l}\text { Post-treatment } \\
1^{\text {st }} \text { month }\end{array}$ & $\begin{array}{l}\text { Post-treatment } \\
2^{\text {nd }} \text { month }\end{array}$ & $\begin{array}{l}\text { Post-treatment } \\
3^{\text {rd }} \text { month }\end{array}$ & $\begin{array}{l}\text { Post-treatment } \\
6^{\text {th }} \text { month }\end{array}$ & \\
\hline Groups & Mean \pm SD & Mean \pm SD & Mean \pm SD & Mean \pm SD & Mean \pm SD & $p^{*}$ \\
\hline \multicolumn{7}{|l|}{ USG } \\
\hline Exercise & $4.6 \pm 0.5$ & $4.6 \pm 0.5$ & $4.6 \pm 0.5$ & $4.5 \pm 0.5$ & $4.5 \pm 0.5$ & 0.121 \\
\hline ESWT+Exercise & $4.7 \pm 0.5$ & $4.7 \pm 0.5$ & $4.6 \pm 0.5$ & $4.6 \pm 0.5$ & $4.6 \pm 0.5$ & 0.632 \\
\hline PRP+Exercise & $4.7 \pm 0.5$ & $4.7 \pm 0.5$ & $4.6 \pm 0.6$ & $4.6 \pm 0.7$ & $4.5 \pm 0.7$ & 0.255 \\
\hline
\end{tabular}

\begin{tabular}{|c|c|c|c|c|c|c|c|c|}
\hline \multirow[b]{4}{*}{ Roles and Maudsley Score } & tre & t $(\mathrm{M}$ & $\begin{array}{l}\text { LE } 5 \\
\text { 6) }\end{array}$ & ison & & & \multirow[b]{4}{*}{$p$} & \multirow[b]{4}{*}{ Post-hoc } \\
\hline & \multicolumn{6}{|c|}{ Groups } & & \\
\hline & \multicolumn{2}{|c|}{ Exercise } & \multicolumn{2}{|c|}{ ESWT+Exercise } & \multicolumn{2}{|c|}{ PRP+Exercise } & & \\
\hline & $\mathrm{n}$ & $\%$ & $\mathrm{n}$ & $\%$ & $\mathrm{n}$ & $\%$ & & \\
\hline \multicolumn{9}{|l|}{ Post-treatment $6^{\text {th }}$ month } \\
\hline Excellent & 6 & 25 & 12 & 48 & 14 & 56 & \multirow{4}{*}{0.033} & \multirow{4}{*}{$0.031 \dagger$} \\
\hline Good & 8 & 33.3 & 6 & 24 & 8 & 32 & & \\
\hline Acceptable & 8 & 33.3 & 5 & 20 & 3 & 12 & & \\
\hline Poor & 2 & 8.3 & 2 & 8 & 0 & 0 & & \\
\hline
\end{tabular}

\section{DISCUSSION}

In the present study, we investigated whether there was a significant difference among the effectiveness of exercise, ESWT and PRP treatments on pain relief, function and muscle strength in the patients with LE. We found a significant improvement in all measurements in all three groups, although better outcomes were achieved in the PRP+Exercise group regarding the measurements related to both pain relief and functionality.

In the light of literature, the number of the studies conducted on the regenerative therapies in the treatment of chronic LE has been progressively increasing by clarification of the pathogenesis of the disease. The use of regenerative therapies in the treatment of LE started at the end of 1990s. ${ }^{[22]}$ However, there is still no consensus on the superiority of these therapeutic modalities among others and related treatment protocols, since they are newly introduced and the studies are conducted with different methodologies.

Alessio-Mazzola et al. ${ }^{[23]}$ compared the efficacy of a single-dose PRP injection and three to four sessions of ESWT per week in patients with chronic LE. They found a statistically significantly higher improvement with respect to VAS, QuickDASH, and PRTEE scores at the end of two years in both groups; however, there was no statistically significant difference between the two groups. Besides, the improvement rate in symptoms in the PRP group was found to be statistically significantly higher than the ESWT group. In another study, Othman ${ }^{[24]}$ compared PRP and ESWT treatments and PRP group demonstrated a statistically significantly higher improvement than ESWT group in terms of both VAS and DASH scores. In our study, a statistically significant improvement in the VAS activity scores was found in all three groups starting from the first month. At six months of follow-up, there was a statistically significant difference in the VAS activity scores at six months compared to baseline between the PRP+Exercise and Exercise groups. With respect to the DASH scores, however, there was no statistically significant difference between the PRP and ESWT groups, whereas a statistically significant difference was found between the PRP+Exercise and Exercise groups. According to the inter-group comparisons 
in terms of PRTEE scores, the PRP+Exercise group showed a statistically significant higher improvement compared to the other two groups (Exercise and ESWT+Exercise).

The data obtained from the studies which compared ESWT with placebo and other treatment methods reveal controversial outcomes regarding the role and effectiveness of ESWT in the treatment of LE. Lefevre et al. ${ }^{[25]}$ reported that there was no strong and consistent evidence of the fact that ESWT contributed to an improvement in LE. On the other hand, Stasinopoulos et al. ${ }^{[26]}$ reported that further well-designed quality studies were needed to draw more reliable conclusions. In addition, comparison and standardization of the various study outcomes are difficult, since different devices are used, shock waves are produced by different mechanisms, and therapy is applied via different doses with varying energy intensities. ${ }^{[27]}$

The grip strength is reduced secondary to pain in the patients with LE, and assessment of grip strength is an objective indicator used in follow-up of the treatment. The measurements of grip strength in the evaluation of treatment efficacy are usually performed at a variety of test positions using different instruments in those patients. ${ }^{[28,29]}$ Dingemanse et al. ${ }^{[30]}$ analyzed three studies on ESWT in their review (totally 455 patients) and concluded that ESWT had no effect on grip strength. However, Notarnicola et al. ${ }^{[31]}$ measured grip strength with a software coordinated with a dynamometer and emphasized that grip strength decreased after treatment in their one-month follow-up study and that this condition might have resulted from the increased tone in the painful muscles. In our study, an increased grip strength was observed in both extension and flexion positions in all three groups, while the ESWT+Exercise group demonstrated a lower increase, particularly in the flexion position than the other two treatment groups and a statistically significant difference was found compared to the PRP+Exercise group. In a systematic review in which the outcomes of patients with LE performing strengthening exercises were analyzed, an improvement was noted with respect to pain, functionality, and handgrip strength in all the treatment groups involving eccentric exercise. ${ }^{[32]}$ In our study, stretching and eccentric strengthening exercises were performed for three months in all three groups. The exercise group revealed a significant improvement in terms of VAS, PRTEE, DASH scores and grip strengths of hand and fingers, consistent with this review.
A study conducted to evaluate the effect of exercise on grip strength compared the stretching and eccentric exercises given as a home-based exercise program for 12 weeks and a higher increase was found in grip strength in the eccentric exercise group, compared to the stretching group. ${ }^{[33]}$ Similarly, a remarkable improvement was observed in pain relief, muscle strength, and functional status in the eccentric exercise group in another study. ${ }^{[34]}$ In the current study, hand and finger grip strengths significantly increased in all three groups, since they were given both stretching and eccentric strengthening exercises, while the PRP+Exercise group was found to be superior to both Exercise and ESWT+Exercise groups in terms of the handgrip strength. This result was correlated with improvement in the VAS activity scores of the PRP+Exercise group.

Finger grip strength is not a commonly used assessment method for LE, as only extensor muscle groups are attached to the lateral epicondyle. However, we found a similarity between the results of handgrip strength and this test which we performed to investigate to what extent finger grip strength was affected in the patients with LE. Based on the results, we can speculate that LE affects weak grip strength of the hand.

In recent years, common extensor tendon thickness measurements by USG is commonly used in the literature for diagnosis and follow-up of LE. Common extensor tendon thickening is expected in the course of LE, whereas a reduction in tendon thickness is anticipated with treatment. This alteration can be measured through USG; however, detection of a reduction in tendon thickness that can be accepted as clinically significant is difficult. No significant difference was seen on USG in terms of common extensor tendon thickness in some studies, while some others showed an increase in the tendon thickness. ${ }^{[9,35,36]}$ In our study, no significant difference was found at the post-treatment sixth month compared to baseline in all three groups. Further long-term studies for at least six months are required to assess accurately a chronic change such as tendon thickening by USG.

The lack of a placebo group which did not receive treatment due to the ethical issues is the limitation in evaluating the effectiveness of the treatments in our study. In addition, other subscales of PRTEE and DASH were unable to be analyzed. Further large-scale, longterm, prospective, randomized-controlled studies are needed to confirm these findings. Also, the fact 
that the researcher who performed the treatments was not blind to the treatment groups and the patients could not be blinded due to the nature of the treatment protocols are the other factors limiting the strength of the study. The lack of a healthy control group for the USG tendon thickness measurements was also one of the limitations. Although the patients were given a medication follow-up form, the use of pain medication could not be clearly evaluated, as the form was not filled out regularly by the patients. Additionally, the lack of an established standardization for posology of ESWT, preparation process of PRP, and exercise protocol in the literature can be regarded as the other limitations.

In conclusion, our study results showed significant improvements in both pain levels and functionality in all three groups. However, PRP+Exercise group achieved better outcomes regarding pain and functionality. These findings highlight the importance of exercise in the treatment of LE, and the effectiveness of the treatment increases when the exercise is combined with a regenerative therapy. Among the regenerative modalities, PRP seems to be superior to ESWT. However, long-term follow-up studies are needed to establish a firm conclusion on this subject.

\section{Declaration of conflicting interests}

The authors declared no conflicts of interest with respect to the authorship and/or publication of this article.

\section{Funding}

The authors received no financial support for the research and/or authorship of this article.

\section{REFERENCES}

1. Wadsworth TG. Tennis elbow: Conservative, surgical, and manipulative treatment. Br Med J (Clin Res Ed) 1987;294:621-4.

2. Rayan GM, Coray SA. V-Y slide of the common extensor origin for lateral elbow tendonopathy. J Hand Surg Am 2001;26:1138-45.

3. Oztuna V, Milcan A, Eskandari MM, Kuyurtar F. Konservatif tedaviye dirençli lateral epikondilitlerin perkütan tenotomi ile tedavisi. Acta Orthop Traumatol Turc 2002;36:336-40.

4. Clinton RE, Murthi A. Lateral epicondylitis. Current Orthopaedic Practice 2008;19:612-5.

5. Peterson M, Butler S, Eriksson M, Svärdsudd K. A randomized controlled trial of exercise versus wait-list in chronic tennis elbow (lateral epicondylosis). Ups J Med Sci 2011;116:269-79.

6. Kochar M, Dogra A. Effectiveness of a specific physiotherapy regimen on patients with tennis elbow. Physiotherapy 2002;88:333-41.
7. Fedorczyk JM. Tennis elbow: Blending basic science with clinical practice. J Hand Ther 2006;19:146-53.

8. Wang CJ. Extracorporeal shockwave therapy in musculoskeletal disorders. J Orthop Surg Res 2012;7:11.

9. Gündüz R, Malas FÜ, Borman P, Kocaoğlu S, Özçakar L. Physical therapy, corticosteroid injection, and extracorporeal shock wave treatment in lateral epicondylitis. Clinical and ultrasonographical comparison. Clin Rheumatol 2012;31:807-12.

10. Wang CJ. An overview of shock wave therapy in musculoskeletal disorders. Chang Gung Med J 2003;26:220-32.

11. Paoloni J, De Vos RJ, Hamilton B, Murrell GA, Orchard J. Platelet-rich plasma treatment for ligament and tendon injuries. Clin J Sport Med 2011;21:37-45.

12. Rees JD, Wilson AM, Wolman RL. Current concepts in the management of tendon disorders. Rheumatology (Oxford) 2006;45:508-21.

13. Rees JD, Maffulli N, Cook J. Management of tendinopathy. Am J Sports Med 2009;37:1855-67.

14. Nguyen RT, Borg-Stein J, McInnis K. Applications of platelet-rich plasma in musculoskeletal and sports medicine: An evidence-based approach. PM R 2011;3:226-50.

15. Capan N, Esmaeilzadeh S, Oral A, Basoglu C, Karan A, Sindel D. Authors' response to a letter to the editor on radial extracorporeal shockwave in the management of lateral epicondylitis. Am J Phys Med Rehabil 2018;97:e11-e12.

16. Fess EE. Grip strength. In: Casanova JS, editor. Clinical Assessment Recommendations. 3rd ed. Chicago, IL: American Society of Hand Therapists; 1992. p. 41-5.

17. Wuori JL, Overend TJ, Kramer JF, MacDermid J. Strength and pain measures associated with lateral epicondylitis bracing. Arch Phys Med Rehabil 1998;79:832-7.

18. Düger T, Yakut E, Öksüz Ç, Yörükan S, Bilgütay BS, Ayhan Ç, et al. Reliability and validity of the Turkish version of the Disabilities of the Arm, Shoulder and Hand (DASH) questionnaire. Fizyoterapi Rehabilitasyon 2006;17:99-107.

19. Altan L, Ercan I, Konur S. Reliability and validity of Turkish version of the patient rated tennis elbow evaluation. Rheumatol Int 2010;30:1049-54.

20. Levin D, Nazarian LN, Miller TT, O'Kane PL, Feld RI, Parker L, et al. Lateral epicondylitis of the elbow: US findings. Radiology 2005;237:230-4.

21. Roles NC, Maudsley RH. Radial tunnel syndrome: Resistant tennis elbow as a nerve entrapment. J Bone Joint Surg [Br] 1972;54:499-508.

22. Mishra A, Pavelko T. Treatment of chronic elbow tendinosis with buffered platelet-rich plasma. Am J Sports Med 2006;34:1774-8.

23. Alessio-Mazzola M, Repetto I, Biti B, Trentini R, Formica M, Felli L. Autologous US-guided PRP injection versus US-guided focal extracorporeal shock wave therapy for chronic lateral epicondylitis: A minimum of 2-year followup retrospective comparative study. J Orthop Surg (Hong Kong) 2018;26:2309499017749986.

24. Othman AMA. Treatment of chronic lateral epicondylitis: Platelet rich plasma versus extra-corporeal shock wave therapy. Open Journal of Orthopedics 2014;4:77-83. 
25. Lefevre F. Extracorporeal shock wave treatment for chronic tendinitis of the elbow (lateral epicondylitis). Technol Eval Cent Assess Program Exec Summ 2005;19:1-23.

26. Stasinopoulos D, Stasinopoulou K, Johnson MI. An exercise programme for the management of lateral elbow tendinopathy. Br J Sports Med 2005;39:944-7.

27. Spacca G, Necozione S, Cacchio A. Radial shock wave therapy for lateral epicondylitis: A prospective randomised controlled single-blind study. Eura Medicophys 2005;41:17-25.

28. Pienimäki T, Tarvainen TK, Siira $P$, Vanharanta $H$. Progressive strengthening and stretching exercises and ultrasound for chronic lateral epicondylitis. Physiotherapy 1996;82:522-30.

29. Vicenzino B, Paungmali A, Buratowski S, Wright A. Specific manipulative therapy treatment for chronic lateral epicondylalgia produces uniquely characteristic hypoalgesia. Man Ther 2001;6:205-12.

30. Dingemanse R, Randsdorp M, Koes BW, Huisstede BM. Evidence for the effectiveness of electrophysical modalities for treatment of medial and lateral epicondylitis: A systematic review. Br J Sports Med 2014;48:957-65.
31. Notarnicola A, Quagliarella L, Sasanelli N, Maccagnano G, Fracella MR, Forcignanò MI, et al. Effects of extracorporeal shock wave therapy on functional and strength recovery of handgrip in patients affected by epicondylitis. Ultrasound Med Biol 2014;40:2830-40.

32. Cullinane FL, Boocock MG, Trevelyan FC. Is eccentric exercise an effective treatment for lateral epicondylitis? A systematic review. Clin Rehabil 2014;28:3-19.

33. Svernlöv B, Adolfsson L. Non-operative treatment regime including eccentric training for lateral humeral epicondylalgia. Scand J Med Sci Sports 2001;11:328-34.

34. Croisier JL, Foidart-Dessalle M, Tinant F, Crielaard JM, Forthomme B. An isokinetic eccentric programme for the management of chronic lateral epicondylar tendinopathy. Br J Sports Med 2007;41:269-75.

35. Krogh TP, Fredberg U, Stengaard-Pedersen K, Christensen $\mathrm{R}$, Jensen P, Ellingsen T. Treatment of lateral epicondylitis with platelet-rich plasma, glucocorticoid, or saline: A randomized, double-blind, placebo-controlled trial. Am J Sports Med 2013;41:625-35.

36. Clarke AW, Ahmad M, Curtis M, Connell DA. Lateral elbow tendinopathy: Correlation of ultrasound findings with pain and functional disability. Am J Sports Med 2010;38:1209-14. 